\title{
The Challenge of High-Resolution Spectrometer Experiments with Exotic Nuclei
}

Hans Geissel ${ }^{1,2}$, John S. Winfield ${ }^{1}$, Georg P.A. Berg ${ }^{3}$, Timo Dickel ${ }^{1,2}$, Bernhard Franczak ${ }^{1}$, Emma Haettner ${ }^{1}$, Naohito Iwasa ${ }^{4}$, Gottfried Münzenberg ${ }^{1}$, Wolfgang R. Plaß ${ }^{1,2}$,

Christoph Scheidenberger ${ }^{1,2}$, Helmut Weick ${ }^{1}$, Martin Winkler ${ }^{1}$, Mikhail Yavor ${ }^{5}$

1. GSI Helmholtzzentrum, Darmstadt, Germany

2. Justus-Liebig Universität Gießen, Gießen, Germany

3. Department of Physics and JINA, University of Notre Dame, Notre Dame, USA

4. Department of Physics, Tohoku University, Sendai, Japan

5. Institute for Analytical Instrumentation, RAS, St. Petersburg, Russia

Exotic atomic nuclei are short-lived species and are characterized by extreme proton-to-neutron ratios. These nuclei are created in stellar reactions and are keys to understand the abundance of the elements in the universe [1]. Exotic nuclei have quite different properties compared to the well-known species close to the valley of beta stability. This observation makes them essential for the basic understanding of nuclear matter. Nowadays, these rare isotopes can also be produced with modern powerful accelerator facilities at several laboratories worldwide [2] which solve the problems of the inherent small production cross section sections via high primary beam intensities and high energies $[3,4]$. However, the successful production of exotic nuclei is only the first necessary step, the separation from the primary beam and from the abundant contaminants and the measurements of their properties are of equal importance [5].

\section{Spectrometer Basics}

High resolution experiments with energetic heavy ions can in principle be performed in-flight with lateral-dispersive and longitudinal-dispersive electromagnetic spectrometers. Lateral-dispersive systems analyze with magnetic and electric dipole fields and apply multipole fields for focusing and correction of image aberrations [6,7]. A typical lateral-dispersive spectrometer stage, such as it is used in many accelerator based heavy ion laboratories, is shown schematically in Figure 1. A lens system is used at the entrance of a dipole magnet and a second one is placed behind to determine the focal plane conditions. The physical quantity of interest is the momentum resolving power which is mainly determined by the entrance emittance of the ion beam to be analyzed and the illuminated area in the dispersive plane of the dipole magnet. Electric fields can also be applied at low energies (at and below the Coulomb barrier) instead of magnets, but the same statements on the resolving power hold in this case as well. In longitudinal dispersive devices such as ion storage rings [8] and multiplereflection time-of-flight mass spectrometers (MR-TOF-MS) $[9,10]$ the challenge is to achieve isochronous conditions with negligible aberrations of the higher-order optics. In general, one can achieve a higher resolving power with the longitudinal-dispersive, multi-path devices compared to the lateraldispersive spectrometers. The latter spectrometers may be applied as in-flight separators for nuclear reaction products and usually consist of a combination of several dispersive stages. The advantage of these spectrometers is the superior spatial isotopic separation of rare isotopes from the intense primary beam and the abundant contaminants. Space-charge problems are of minor importance for such lateral-dispersive spectrometers. They can have an overall maximum momentum resolving power $(p / \Delta p)$ of several $10^{4}[11,12]$, whereas storage rings and MR-TOF-MS systems can achieve more than 50 times higher resolving powers. In lateral spectrometers, the spatial deflections and resolving power are measured by position detectors, whereas in storage rings and MR-TOF-MS systems the observables are the revolution frequency or the time of flight for many turns $(T)$. The mass resolving power $(\mathrm{m} / \Delta m)$ is determined by $T$ and its experimental uncertainty $\Delta T$. 


\section{a) Lateral-Dispersive Spectrometer (Single-Path System)}

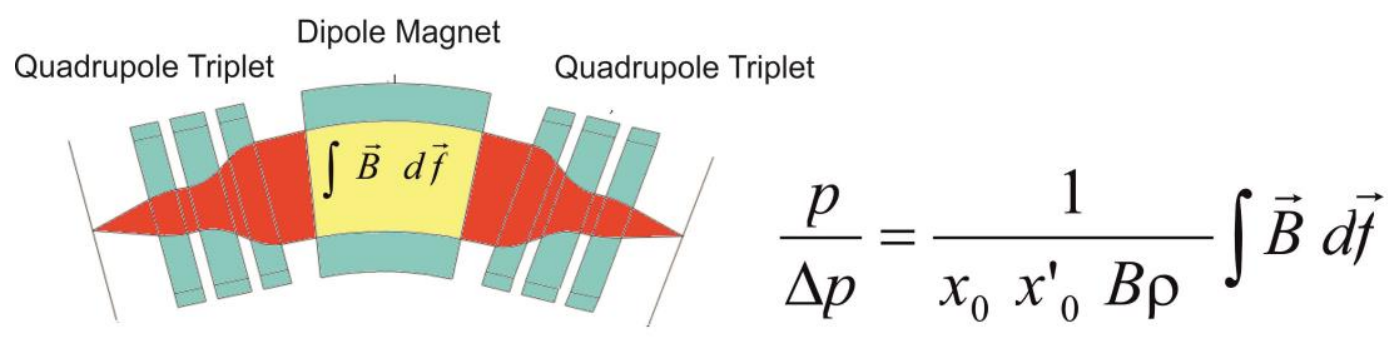

b) Longitudinal-Dispersive Spectrometer (Multi-Path System)

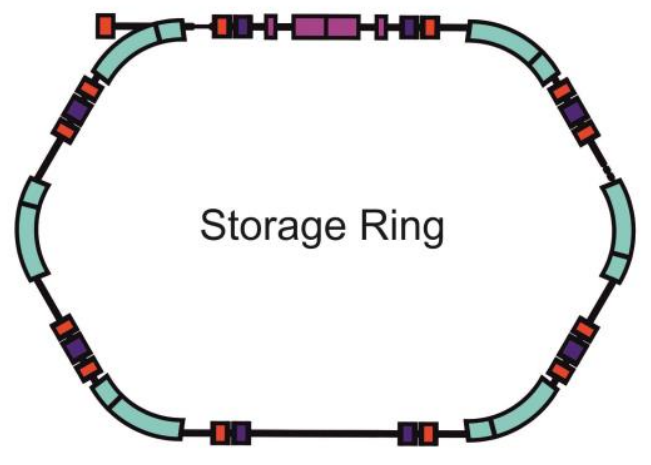

MR-ToF-MS

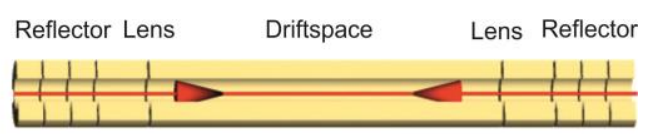

$\frac{m}{\Delta m}=\frac{T}{2 \Delta T}$

Figure 1. Principles of lateral- and longitudinal-dispersive spectrometers that are applied for research of exotic nuclei in many accelerator laboratories. In the example of the magnetic dipole stage (panel a) the momentum resolving power $p / \Delta p$ is determined by the entrance emittance $x_{0} \cdot x_{0}{ }^{\prime}$ and the illuminated field area in the dipole magnet. In longitudinal-dispersive ion-optical devices such as storage rings and multiple-reflection time-of-flight spectrometers (MR-TOF-MS) the resolving power is obtained via frequency and flight time $(T)$ measurements.

\section{Exotic Nuclei, Production and Separation}

In conventional ion-optical systems governed by conservative forces the phase space of the transported ions is preserved according to the well-known Liouvillian theorem. This is not the case in modern spectrometer experiments with exotic nuclei, where atomic and nuclear interactions in matter are applied within the ion-optical system [13]. This non-Liouvillian optics requires special instrumentation and techniques to enable high-resolution measurements.

Exotic nuclides produced and separated in-flight have an inevitable large phase space due the stochastic nuclear collision processes in the production target. The relative momentum and angular spread of medium mass rare isotopes created in projectile fragmentation are in the $10^{-2}$ and in the several milliradian ranges, respectively. The corresponding emittance growth of fission products is by roughly a factor of 4 larger, Figure 2 . In addition to the nuclear reactions comes the contribution of atomic collisions for the detectors, target and degraders. The latter two material layers are relatively thick, being up to $10 \%$ of the atomic range of the incident primary beam in the target material and about $50 \%$ of the atomic range for the selected fragment in the degrader material $[16,17,18]$. From these kinematical properties it becomes clear that high-resolution spectrometer measurements with exotic nuclei are a great challenge.

A major goal is to measure subtle kinematical properties such as the momentum distribution of valence nucleons after secondary reactions with much higher resolution than the inevitable incident momentum or angular spread of the primary fragments. This may be achieved by the use of dedicated ion-optical systems such as energy-loss (dispersion-matched) spectrometers and isochronous sys- 
tems, or methods which reduce the incident phase space by cooling and energy bunching. Recent experiments [19,20] with the fragment separator FRS [21] and the storage-cooler ring ESR [22] have demonstrated the success of these efforts and are the base for the next generation facility Super-FRS $[18,23]$, which will provide an even wider discovery potential. Analogous results can be obtained with the present and planned facilities in other heavy ion laboratories.
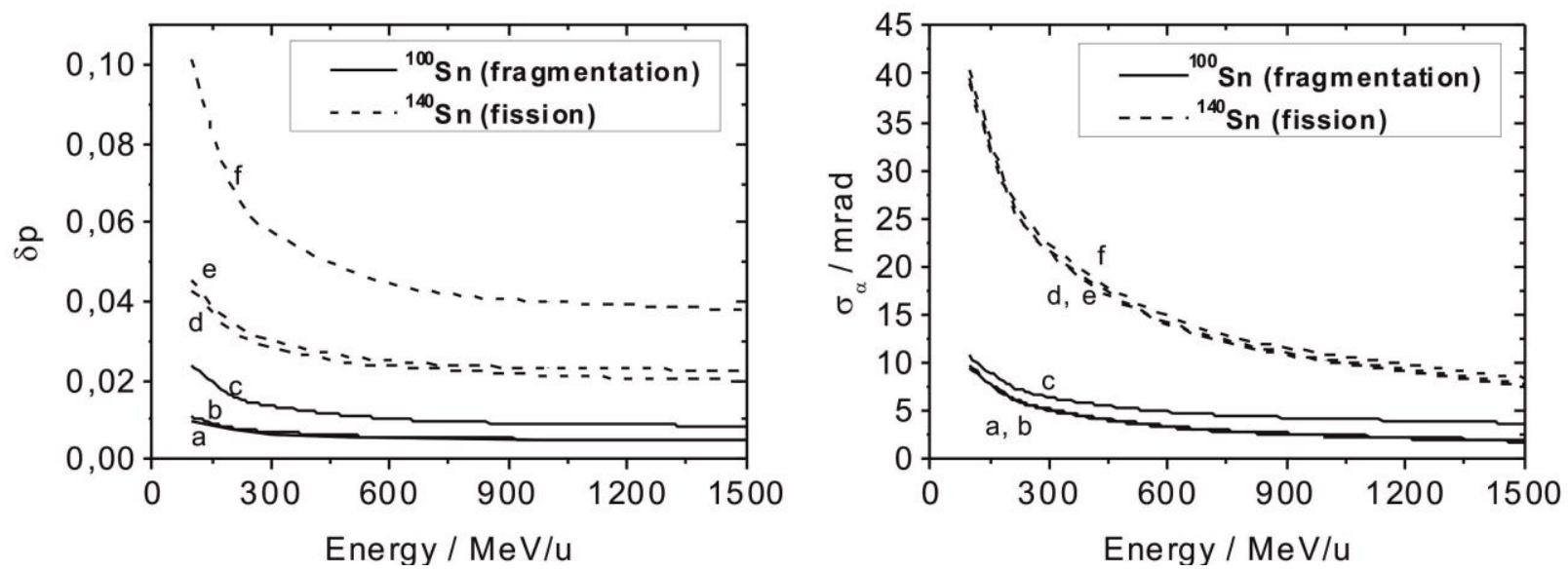

Figure 2. Calculated $[14,15]$ relative momentum and angular spread of ${ }^{100} \mathrm{Sn}$ and ${ }^{140} \mathrm{Sn}$ exotic nuclides created with ${ }^{124} \mathrm{Xe}$ and ${ }^{238} \mathrm{U}$ primary beams, respectively. The contributions of the nuclear reactions (curves labeled a) and the convolution with the atomic interaction in thick Be targets $\left(\mathrm{t} / \mathrm{R}_{\mathrm{p}}=\right.$ $0.1)(b)$ and penetrating $\mathrm{Al}$ degraders of $\left(\mathrm{d} / \mathrm{R}_{\mathrm{f}}=0.5\right)(\mathbf{c})$ are shown separately.

\section{Experiments with Energy Compression}

\section{a. Lateral-dispersive Spectrometer with a Mono-energetic Degrader}

In Figure 3 the symmetric direct branch of the FRS is depicted schematically with its 4 magnetic dipole stages including quadrupole lenses and hexapole magnets in front of and behind each $30^{\circ}$ dipole magnet. The optical system has a total length of about $75 \mathrm{~m}$ and includes besides the magnetic elements focal-plane detectors for diagnostics and particle identification. In the standard operation of isotopic separation, the ion-optical system of the FRS is achromatic from F0 to F4 and a shaped energy degrader is used which preserves the achromatic condition [21]. This operating mode has the best performance for spatial isotopic separation in-flight and is also preferable for experiments with the combination with the ESR. However, there are experiment classes where an efficient energy compression has priority because the separated exotic nuclides must be completely stopped (thermalized) in a helium-filled $\left(5 \mathrm{mg} / \mathrm{cm}^{2}\right)$ cryogenic (70-100 K) stopping cell (CSC) [24], i.e., the normally large range straggling of the fragments due to their velocity spread must be strongly reduced with a so-called mono-energetic degrader [25]. A mono-energetic degrader has a special shape which matches the optical dispersion of the FRS in such a way that the energy loss is larger for the faster ions so that the energy of all ions after penetration is the same all over the dispersive plane. An example of the calculated [26] phase-space modifications caused by a mono-energetic degrader at the central focal plane of the FRS is shown in Figure 4. 


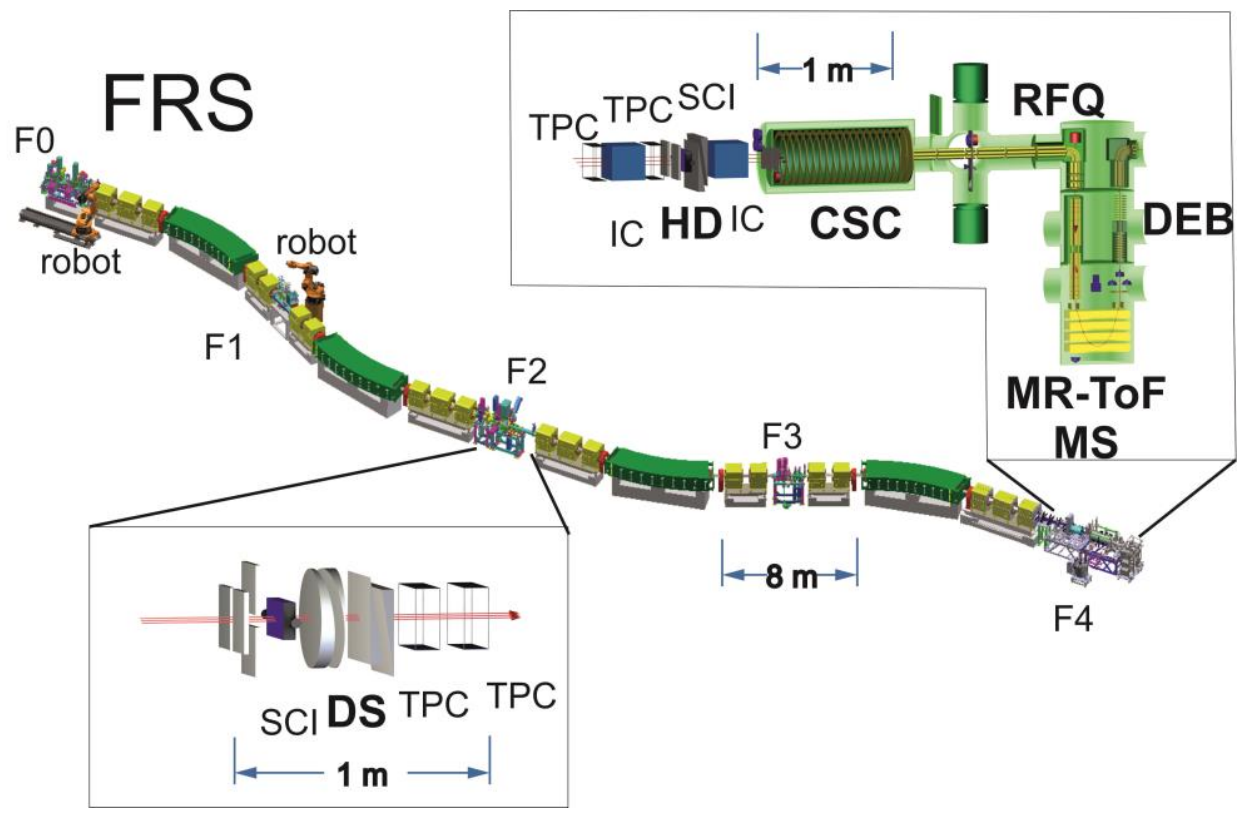

Figure 3. Production (F0), spatial separation in-flight (F0-F4) and energy compression (F2) of exotic nuclei with the separator-spectrometer FRS [21]. The complete particle identification with the FRS has been provided by time-of-flight (SCI), position (TPC) and energy-deposition (IC) measurements at the focal planes F2 and F4. The selected fragment beam is completely stopped and thermalized in the cryogenic gas cell (CSC) [24]. The energy compression is performed with a mono-energetic degrader system (MD) placed at the central dispersive focal plane of the FRS. The final slowing down in front of the CSC is done with a homogenous variable degrader (HD). The thermalized exotic nuclei extracted from the CSC with dc and Rf fields are transported and analyzed through buffer-gas filled radio frequency quadrupoles (RFQ) before they are injected in the MR-TOF-MS.

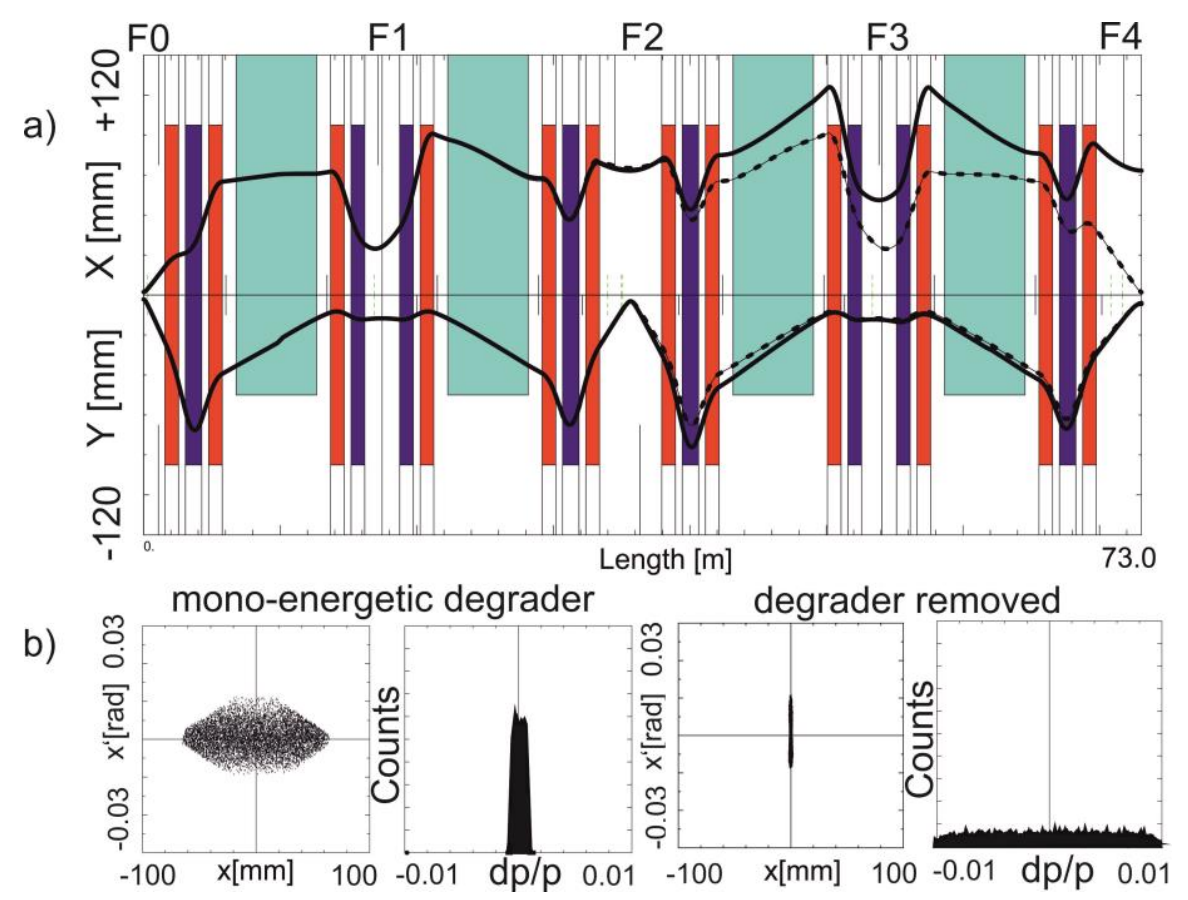

Figure 4. Calculated [26] phase-space modifications caused by a mono-energetic degrader at the central focal plane of the FRS. The fragments emerging from the target (F0) have a $2 \%$ relative momentum spread. Panel a): Envelopes in x- and y-direction with (full lines) and without the degrader (dashed lines). Panel b): Calculated momentum, position and angular distributions at the final focal plane with and without the mono-energetic degrader. 
The achievable compression of the range straggling is strongly dependent on the momentum resolving power $(p / \Delta p)$ of the optical system directly in front of the mono-energetic degrader (MD). When the MD is placed at the central focal plane F2 the resolving power is about 2300 for an initial spot size of $+/-1.5 \mathrm{~mm}$ at the production target. The minimum range straggling $\left(\sigma_{\mathrm{R} 0}\right)$, which is solely due to the stochastic atomic collisions of the ions during their slowing down in matter, can only be achieved for an ideal incident heavy ion beam with zero energy spread. For this case $\sigma_{\mathrm{R} 0}$ is about $1 \%$ of the corresponding mean range valid for 100-1000 MeV/u ions in amorphous matter [15]. With the FRS we could approach the limit of $\sigma_{\mathrm{R} 0}$ by a factor of 1.2 in recent experiments.

\section{b. Electron Cooling of Exotic Nuclei in a Storage Ring}

In an ion storage-cooler ring the injected hot (large emittance) circulating fragment beam is merged with a cold and constantly-refreshed electron beam of low emittance in a several meter long, straight section of the circumference. The stored heavy ions loose energy in collisions with the cold electrons until the relative velocity becomes zero. In this way the momentum spread of the incident hot fragment beam can be reduced to $10^{-5}-10^{-7}$ depending on the intensity of the stored beam [27]. Although electron cooling is an elegant experimental method, the cooling time limits the access to very shortlived exotic nuclei. The electron cooling time $\left(\tau_{\mathrm{e}}\right)$ strongly depends on the initial velocity spread $(\Delta \mathrm{v})$ of the injected fragments $\left(\tau_{\mathrm{e}} \propto \Delta \mathrm{v}^{-3}\right)$. With such electron-cooled fragment beams one can ideally perform high-resolution experiments [8]. Accurate mass measurements have been successfully carried out in the past for a large number of isotopes with previously unknown experimental masses [28,20]. In these experiments also new isotopes have been discovered. The electron-cooling times were 5-10 s. The overall cooling time can be shortened via stochastic pre-cooling [29], especially for intense ion beams with large emittance. Stochastic cooling reduces the longitudinal velocity spread in about $3 \mathrm{~s}$ below $10^{-3}$ which speeds up the subsequent electron cooling to a few seconds.

\section{c. The Dynamic Energy-Buncher at Low Energies}

The slowing down with electromagnetic retarding fields is quite different from the process of ion penetration through matter. When energetic ions are decelerated in static electric fields, only the vector component that is opposite to the retarding field is reduced. Therefore, the angular and energy straggling would be not reduced. However, in some experiments at low energy systems it is essential to reduce the straggling as well. In the experimental scenarios under consideration, such a requirement is met by an accumulation trap within the MR-TOF-MS, where a high efficiency of ion collection is needed in front of buffer-gas cooling sections.

The experimental solution can be a dynamic energy buncher (DEB) [30,31] which is schematically shown in Figure 5. The setup starts from a time focus in a drift space in front of a ring-electrode system which applies a homogeneous electric retarding field. When the ion bunch with a large velocity spread of about $50 \mathrm{eV}$ has completely entered the electrode system, the electric field is switched off. To first order, the velocity spread can be reduced to about $1 \mathrm{eV}$ if the relation between the incident mean kinetic energy $K_{0}$, the drift length $d$ and the electric field $E$ is $K_{0}=q E \cdot d / 2$. This condition means that the velocity distribution of the emerging ion bunch from the DEB is to first order independent of the velocity spread of the incident ions starting at the time focus. The condition can also be interpreted in a way that ions with a charge state $q$ can be stopped in the retarding field after they have travelled a distance $d / 2$. After the energy spread is dramatically reduced in the DEB, the ions can be further decelerated to the required maximum mean energy for a gas-filled RFQ by means of an additional retarding field - see the experimental setup and calculated spectra in Figure 5. The calculation demonstrates that the DEB with the described constant field is characterized by a systematic quadratic flight-time aberration, see insertion. 

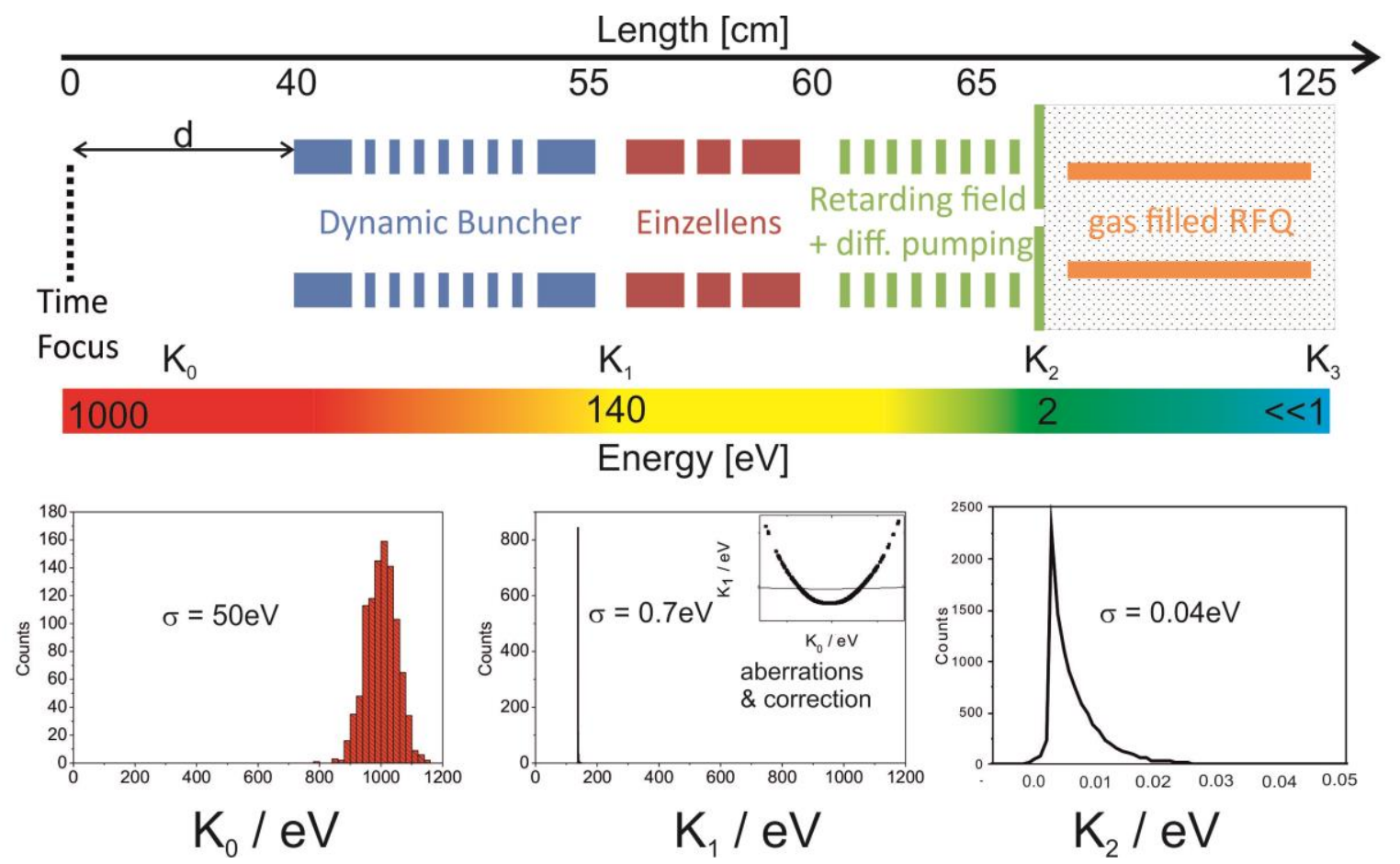

Figure 5. Deceleration and energy compression with a dynamical electrostatic buncher. The electric field strength is switched off after a chosen time delay corresponding to the flight time of the incident beam from the time focus. An incident $1000 \mathrm{eV}^{140} \mathrm{Sn}^{1+}$ beam with an energy spread of $50 \mathrm{eV}$ is decelerated to a mean energy of $160 \mathrm{eV}$ and a spread of $0.7 \mathrm{eV}$. A second retarding field is applied before the bunch is cooled in a gas-filled radio-frequency quadrupole (RFQ).

This dependence can be easily removed by adding a small quadratic component to the retarding field with the result that the energy spread of the energy distribution $\left(K_{1}\right)$ is improved by roughly a factor of 10 . However, in practice the spread of the time focus is the limiting factor, i.e. already a time spread of $5 \mathrm{~ns}$ exceed the second order aberration of the DEB. Of course, one can improve the resolving power also by increasing $d$ which would also change the other dimensions in the present setup. The results of our detailed investigation of the DEB performance will be published in a separate paper.

\section{d. Buffer-Gas Cooling}

High resolving powers can be achieved in low-energy traps in a similar way as described for the high energy experiments, namely either by applying conditions that the ion-optical image condition at a final focal plane are independent of the initial energy spread or the phase is reduced vial cooling processes [32]. A low energy ion beam with a maximum kinetic energy of about $5 \mathrm{eV}$ can be captured and cooled in a gas-filled radiofrequency quadrupole (RFQ). The captured and stored ions can via atomic collisions reach in a short time (a few $\mathrm{ms}$ ) the temperature of the gas. In elastic atomic collisions the ions transfer energy to the lighter atoms in the RFQ until equilibrium is reached. Typically helium gas at a pressure of $1 \mathrm{~Pa}$ at room temperature is used in a 0.3 to $1 \mathrm{~m}$ long RFQ which is differentially pumped. In many aspects the buffer gas cooling is quite similar to the described electron cooling in a storage ring but the buffer-gas cooling is much faster (see above). In simulations with the program ITSIM [33] we have determined the maximum incident kinetic energy $K_{2}$ as a function of the helium areal density. As an example if it is $2 \mathrm{eV}$ the required areal density of the helium buffer gas is about $5 \cdot 10^{-5} \mathrm{mg} / \mathrm{cm}^{2}$. This result is implemented in the illustrated experimental setup in Figure5. 


\section{Dispersion-matched and Isochronous Spectrometers}

\section{a. The dispersion-matched sections with the Super-FRS}

Another solution to perform high-resolution measurements with an incident ion beam characterized by a large phase space is to use dedicated ion-optical spectrometer modes which circumvent the influence of the incident large momentum and angular spread at the observables at final focal plane. The so-called "energy-loss" or "dispersion-matched" spectrometers are such tools which can be applied for this task [12].

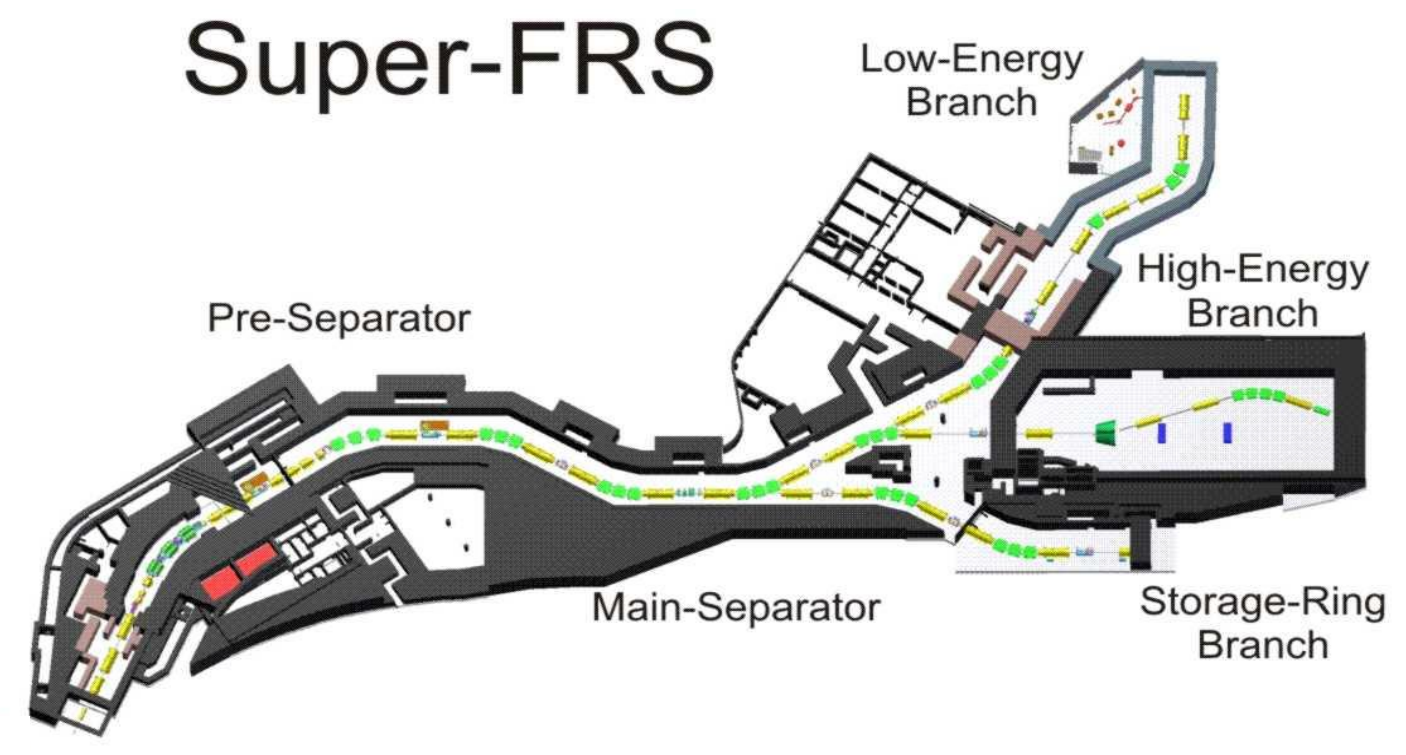

Figure 6. Layout of the in-flight separator Super-FRS [18] and its three experimental branches at FAIR.

The Superconducting FRagment Separator (Super-FRS), presently under construction, is a powerful in-flight facility which will provide-spatially separated isotopic beams up to elements of the heaviest projectiles. It is superior to the present FRS due to the incorporation of more separation stages and larger magnet apertures [18]. The Super-FRS, based on the results and experience of present FRS, has a Pre- and a Main-Separator unit and three branches, see Figure 6. It has a larger acceptance and more separation stages, Pre- and Main-Separator, than the present FRS facility. The gain factor in transmission is more than one order of magnitude for fragments far from the mass number of the projectiles. In addition, it has also a larger maximum magnetic rigidity $\left(B \rho_{\max }=20 \mathrm{Tm}\right)$ to improve the separation quality by suppression of different charge states for the heaviest fragments, i.e. at these high velocities the ions of all elements emerge fully ionized from the targets and degraders. The different ion-optical stages of the Super-FRS can be independently operated in the standard separator mode but can also be combined to have dispersion-matched sub-sections for dedicated experiments, see Figure 7.

Recently, we have calculated and proposed such a combined high-resolution system for the LowerEnergy-Branch (LEB) [12]. The magnetic elements of the Main-Separator and the Energy Buncher have been combined to a high-resolution dispersion-matched spectrometer. For experiments at the maximum magnetic rigidity $\mathrm{B} \rho_{\max }$ of $20 \mathrm{Tm}$, the Main-Separator can be used as an energy-loss spectrometer. The optical calculation for this mode is presented in Figure 8. 

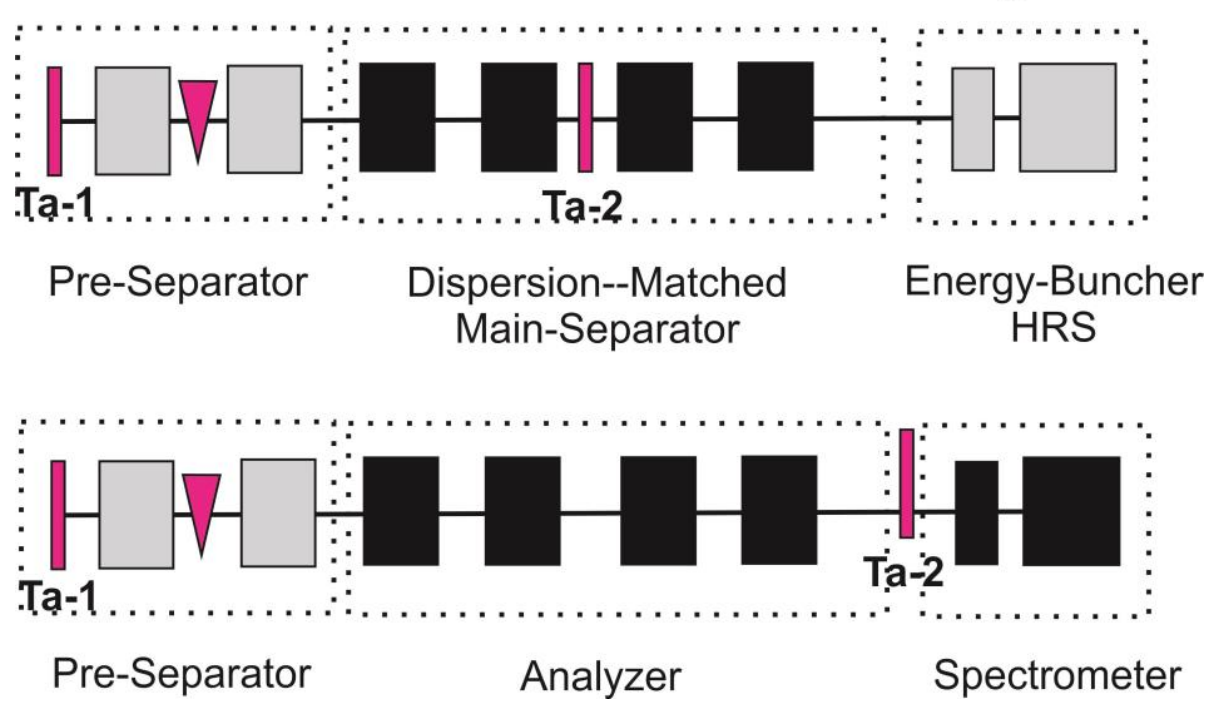

Figure 7. The ion-optical system of the Super-FRS can be combined to dispersion-matched spectrometers (schematically shown in black). The Main-Separator parts can be used as an energy-loss spectrometer up to $20 \mathrm{Tm}$. The matching of the Main-Separator with the Energy-Buncher of LEB or the High-resolution spectrometer (HRS) of the HEB have the advantage of a higher resolving power but are restricted to $7 \mathrm{Tm}$ and $15 \mathrm{Tm}$, respectively. The high-resolution studies are performed for reactions in the secondary target Ta-2.

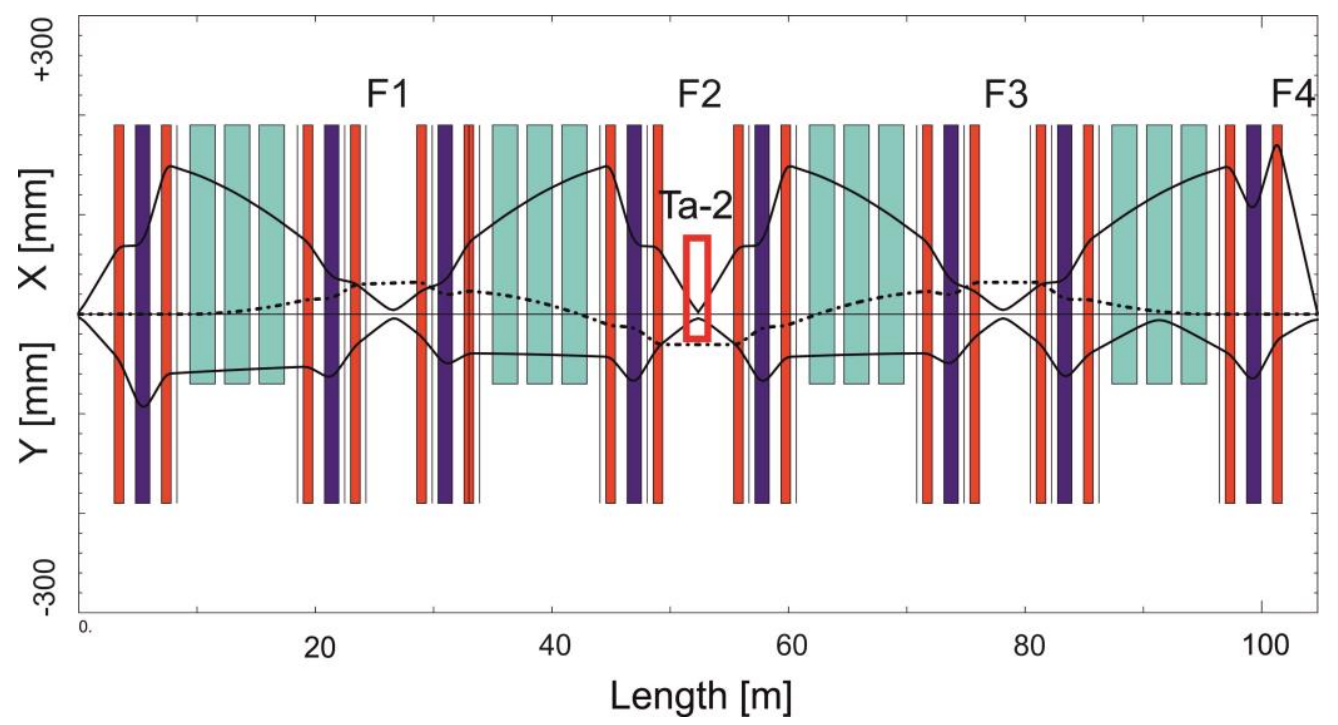

Figure 8. Ion-optical layout of the dispersion-matched Main-Separator of the Super-FRS. The calculated envelopes [26] correspond to an emittance of $\varepsilon_{\mathrm{x}, \mathrm{y}}=40 \pi \mathrm{mm} \mathrm{mrad}$. The dispersion line (dashed) represents a momentum deviation of $0.5 \%$.

The resolving power can be doubled if the EB is matched with the Main-Separator, however, then $\mathrm{B} \rho_{\max }$ of the fragments is limited to $7 \mathrm{Tm}$. A dispersion-matched system with $\mathrm{B} \rho_{\max }$ in-between would be the matching of the Super-FRS with the planned High-Resolution-Spectrometer in the High-Energy Branch (HEB).

\section{b. The Isochronous Storage Rings}

The duration of the electron-cooling process is a basic limitation for the access of very short-lived stored nuclei in mass and lifetime experiments. This restriction can be circumvented by operating the storage ring in the isochronous mode [34]. In this special ion-optical mode the revolution time is in- 
dependent of the velocity spread because the faster ions of each isotope are guided on longer trajectories to preserve a constant revolution time. In first isochronous experiments a mass resolving of better than $10^{-5}$ has been reached [35]. However, the isochronous condition is strictly fulfilled only for one selected mass-to-charge $(\mathrm{m} / \mathrm{q})$ ratio in a narrow $\mathrm{B} \rho$ range. A solution is to measure the $\mathrm{B} \rho$ or the velocity of each fragment in addition to the revolution time [36,37]. With $\mathrm{B} \rho$ tagging from the central focal plane of the FRS a mass resolving power of 200000 (FWHM) over a large $m / q$ range can be achieved.

\section{c. The MR-TOF-MS}

The multiple-reflection time-of-flight mass spectrometer (MR-TOF-MS) [38] is a high resolution isochronous system for mass measurements and spectroscopy of low-energy ( 1 keV) exotic nuclei. The exotic nuclides produced and separated in flight at relativistic energies are slowed down with a mono-energetic degrader system, implanted in the cryogenic stopping cell (CSC) and afterwards, extracted within $\sim 20 \mathrm{~ms}$, analyzed and cooled with a gas-filled RFQ and finally injected into the MR-TOF-MS [39], see section above. In an MR-TOF-MS the exotic nuclides are analyzed in flight time coordinate according to their $\mathrm{m} / \mathrm{q}$ ratio. Ideally the flight-time is independent of their kinetic energy and transverse emittance. The main parts are two cylindrical grid-free electrostatic reflectors $0.9 \mathrm{~m}$ apart. After injection the ions are trapped and travel for a selected number of turns between the electrostatic reflectors before they are extracted and hit the timing detector to generate the mass spectrum. Unlike lateral-dispersive spectrometers, TOF mass analyzers accept the entire mass range without any scanning or restriction by the detector size. An MR-TOF-MS can be used for isobar separation and accurate mass measurements of very short-lived heavy nuclei with half-lives of about $1 \mathrm{~ms}$ or longer, and decay spectroscopy. The main characteristics are given in the table below:

\begin{tabular}{|l|l|}
\hline MR-TOF-MS characteristics [38] \\
\hline Transmission efficiency $70 \%$ & Flight time $10 \mathrm{~ms}$ \\
\hline Repetition rate $400 \mathrm{~Hz}$ & Ion capacity $>10^{6} \mathrm{~s}$ \\
\hline Mass resolving power $6 \cdot 10^{5}$ & Mass accuracy $10^{-7}$ \\
\hline
\end{tabular}

References:

[1] FK Thielemann et al, Prog. Part. Nucl. Phys. 66 (2011), p. 346.

[2] Proceedings EMIS-2012, eds. H. Sakurai et al, Nucl. Instr. Meth. B 317 (2013), p. 187.

[3] J Kurcewicz et al, Phys. Lett. B 717 (2012), p. 371.

[4] T Onishi et al, J. Phys. Soc. Jpn. 79 (2010), 073201.

[5] H Geissel et al, „Encycl. Nucl. Phys. Appl.”, ed. R Stock, (Wiley) (2013).

[6] H Wollnik in "Optics of Charged Particles", (Acad. Press, Orlando).

[7] M Yavor in "Optics of Charged Particle Analyzers" (Acad. Press, San Diego) p. 2009.

[8] B Franzke, H Geissel and G Münzenberg, Mass Spectrom. Rev. 27 (2008), p. 428.

[9] H Wollnik and M Przewloka, J. Mass Spectrom. 96 (1990), p. 267.

[10] WR Plaß, T Dickel and C Scheidenberger, Int. J. Mass Spectrom. 349-350 (2013), p. 134.

[11] T Wakasa et al, Nucl. Instr. Meth. Phys. Res. A 482 (2002), p. 79.

[12] H Geissel et al, Nucl. Instr. Meth. Phys. Res. B 317 (2013), p. 277.

[13] H Geissel et al, Nucl. Instr. Meth. Phys. Res. A 282 (1989), p. 247.

[14] N Iwasa et al, Nucl. Instr. Meth. Phys. Res. B 126 (1997), p. 284.

[15] H Geissel, Program ATIMA, http://web-docs.gsi.de/ weick/atima/.

[16] T Kubo, Nucl. Instr. Meth. Phys. Res. B 204 (2003), p. 97.

[17] G Bollen, Intl. Symposium on Exotic Nuclei, Sochi, Russia (2009), AIP Conf. Proc. 1224

(2010) p. 432. 
[18] H Geissel et al, Nucl. Instr. Meth. Phys. Res. B 204 (2003), p. 71.

[19] R Kanungo et al, Phys. Rev. Lett. 102 (2009), 152501.

[20] L Chen et al, Nucl. Phys. A 882 (2012), p. 71.

[21] H Geissel et al, Nucl. Instr. Meth. Phys. Res. B 70 (1992), p. 286.

[22] B Franzke, Nucl. Instr. Meth. Phys. Res. B 24/25 (1987), p. 18.

[23] B Rubio and T Nilsson, Nuclear Physics News 16 (2006), p. 19.

[24] S Purushothaman et al, Europhys. Lett. 104 (2013), 42001.

[25] JS Winfield et al, Nucl. Instr. Meth. Phys. Res. A 704 (2013), p. 76.

[26] B Franczak in "Computing in Accelerator Design and Operation" eds. W Busse and R Zelazny, (Springer, Berlin) p. 170.

[27] M Steck et al, Phys. Rev. Lett. 77 (1996), p. 3803.

[28] YA Litvinov et al, Nucl. Phys. A 734 (2004), p. 473.

[29] F Nolden et al, Nucl. Instr. Meth. Phys. Res. A 441 (2000), p. 219.

[30] WR Plaß et al, Nucl. Instr. Meth. Phys. Res. B 266 (2008), p. 4560.

[31] M Yavor, accepted for publication by Int. J. Mass Spectrom. 2015.

[32] J Äystö and A Jokinenen, J. Phys. B. Atomic, Molecular, Optical Phys. 36 (2003), p. 573.

[33] WR Plaß et al, Proc. 56 ${ }^{\text {th }}$ ASMS Conf. Mass Spectrometry and Allied Topics, Denver, USA (2008) p. MP 015.

[34] H Wollnik, Nucl. Instr. Meth. A 258 (1987), p. 289.

[35] M Hausmann et al, Nucl. Instr. Meth. Phys. Res. A 446 (2000), p. 569.

[36] H Geissel et al, Hyperfine Interact. 173 (2006), p. 49.

[37] B Sun et al, Nucl. Phys. A 812 (2008), p. 1.

[38] T Dickel, accepted by Nucl. Instr. Meth. Phys. Res. B 2015.

[39] WR Plaß et al, Nucl. Instr. Meth. Phys. Res. B 317 (2013), p. 457. 